\title{
Isotope Effect in the Vibrational Predissociation of van der Waals Molecules: Complexes of Glyoxal with $\mathrm{H}_{\mathbf{2}}$ and $\mathrm{D}_{\mathbf{2}}$
}

\author{
J. A. BESWICK, N. HALBERSTADT, C. JOUVET and B. SOEP \\ Laboratoire de Photophysique Moléculaire du CNRS Bâtiment 213, Université \\ Paris-Sud 91405 Orsay, France
}

(Received March 3, 1982; in final form April 30, 1982)

In this work photodissociation studies of glyoxal- $\mathrm{H}_{2}\left(\mathrm{D}_{2}\right)$ molecules have been conducted in order to analyze the effect of the mass in the vibrational predissociation of van der Waals complexes. The glyoxal- $\mathrm{H}_{2}\left(\mathrm{D}_{2}\right)$ molecules have been formed in a supersonic expansion and photodissociated by means of a tunable dye laser. The final state of the glyoxal fragments has been determined by the analysis of the fluorescence spectrum. A significant isotope effect on the branching ratios for predissociation has been found. An interpretation in terms of a distorted wave diabatic treatment is presented.

The dissociation of van der Waals molecules exhibits a clear cut example of vibrational predissociation which constitutes a particular case of the very important problem of vibrational energy redistribution in polyatomic molecules. ${ }^{1-4}$ We have recently undertaken the study of the photodissociation pathways of van der Waals molecules involving a polyatomic (glyoxal) and a rare-gas atom $\left(\mathrm{He}, \mathrm{Ne}\right.$ or $\mathrm{Ar}$ ), or a diatomic molecule $\left(\mathrm{H}_{2}\right){ }^{3,4}$ Those experiments have shown selective pathways for vibrational predissociation which depend on the complex partner.

In an attempt to further understand the dynamics of the process of

Work supported in part by Université Pierre et Marie Curie, UER 52. 
vibrational predissociation, we have conducted new experiments on glyoxal$D_{2}$ : isotope substitution of $H_{2}$ by $D_{2}$ gives a unique way of changing the reduced mass for the van der Waals vibrational motion without changing the intermolecular potential. The purpose of this note is to present the results of those experiments together with a theoretical interpretation based on a distorted wave diabatic treatment of vibrational predissociation. ${ }^{1}$

The experimental setup has been described elsewhere. ${ }^{3-6}$ In brief, the glyoxal- $\mathrm{H}_{2}\left(\mathrm{D}_{2}\right)$ molecules are formed in a supersonic expansion with $\mathrm{He}$ as a carrier gas, excited by a pulsed tunable dye laser to the $8^{1}$ vibrational level of the $S_{1}{ }^{1} A_{u}$ electronic state of glyoxal and the final state of the glyoxal fragment is determined by analysis of the fluorescence spectrum.

In Figure 1, we present the fluorescence excitation spectrum of glyoxal$\mathrm{H}_{2}$ and glyoxal- $\mathrm{D}_{2}$ molecules. Each vibrational transition of the uncomplexed molecule is accompanied by satellite peaks $(a, b, c, d, e)$. We attribute the (b), (c), (d), (e) peaks to a vibrational progression in the van der Waals mode $(0 \rightarrow 0,1,2,3)$, and the (a) peak to a hot band $(1 \rightarrow 0)$. For glyoxal$\mathrm{H}_{2}$ we have determined $\omega=17 \mathrm{~cm}^{-1}$ and $\omega \mathrm{x}=1.4 \mathrm{~cm}^{-1}$ for the frequency and anharmonicity of the van der Waals mode in the electronic excited state. ${ }^{3}$ If this interpretation is correct we expect for glyoxal- $D_{2}$ a reduction of the frequency by a factor $\eta^{1 / 2}$, where $\eta$ is the ratio between the reduced masses $\eta \equiv \mu$ (glyoxal- $\left.\mathrm{D}_{2}\right) / \mu\left(\right.$ glyoxal- $\left.\mathrm{H}_{2}\right)=1.98$, while the anharmonicity should be reduced by the factor $\eta$. We expect then $\omega=12.2$ $\mathrm{cm}^{-1}$ and $\omega x=0.7 \mathrm{~cm}^{-1}$ for glyoxal- $\mathrm{D}_{2}$. From our results presented in Fig. 1 we get $\omega=12 \mathrm{~cm}^{-1}$ and $\omega x=1 \mathrm{~cm}^{-1}$, which agrees with the above prediction within experimental errors. These results confirm the attribution of the satellite peaks of Figure 1 to a vibration progression in the van der Waals mode.

From the analysis of the fluorescence spectrum, we have determined the final vibrational states of the glyoxal fragment produced by vibrational predissociation, and their branching ratios. The results were the following: a) for glyoxal- $\mathrm{H}_{2}$ complexes, only the zero-point energy level $\mathrm{O}^{\circ}$ is significantly populated. A small amount of fluorescence from the $7^{1}$ mode has been detected. We estimate that the branching ratio $R_{H_{2}}\left(\mathrm{O}^{\circ} / 7^{1}\right)$ is in any case larger than 10 ; b) for glyoxal- $D_{2}$ complexes the amount of fluorescence from $7^{1}$ relative to the $\mathrm{O}^{\circ}$ is much larger. We have determined that starting from the $8^{1}$ level of the complex, $20 \%$ of the glyoxal fragments are produced in the $7^{1}$ level, the other $80 \%$ corresponding to the glyoxal fragment in the $\mathrm{O}^{o}$ state. The branching ratio is accordingly $R_{D_{2}}\left(\mathrm{O}^{o} / 7^{1}\right)=$ 4. The ratio $\mathrm{y} \equiv R_{\mathrm{H}_{2}} / R_{D_{2}}$ is then larger than 2.5. 


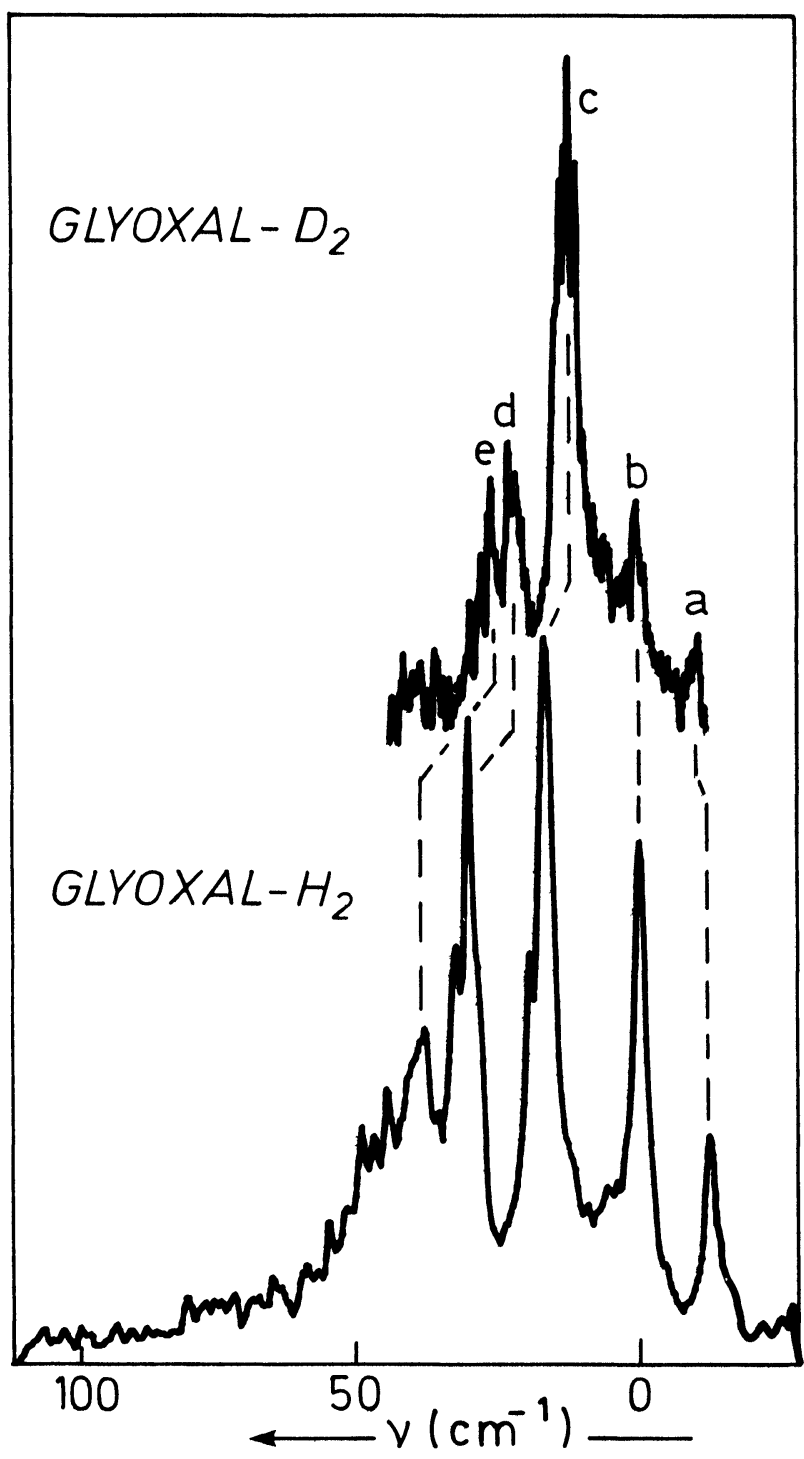

FIGURE 1 State selected ( $\mathrm{O}_{o}^{o}$ emission of glyoxal fragments) fluorescence excitation spectra of glyoxal- $\mathrm{D}_{2}$ (upper trace) and glyoxal- $\mathrm{H}_{2}$ (lower trace), following excitation in the region of the $8_{o}^{1}$ band of glyoxal. Glyoxal was expanded in a mixture of $5 \% \mathrm{D}_{2}$ or $\mathrm{H}_{2}$ in Helium at $15 \mathrm{~atm}$ through a $32 \mu$ nozzle. The peaks a,b,c,d,e are attributed to the same vibrational bands for both complexes, glyoxal- $\mathrm{H}_{2}$ and glyoxal- $\mathrm{D}_{2}$. 
This result can be understood in terms of the distorted wave diabatic treatment for vibrational predissociation in polyatomic systems ${ }^{1,4,7}$ In this model, the bound and dissociative states of the complex are described by diabatic functions:

$$
\psi(\mathrm{R}, \mathrm{r})=\theta(\mathrm{R}) \varphi \mu(\mathrm{r})
$$

where $r$ stands for all the internal coordinates of the glyoxal molecule, and $R$ denotes the distance between the centers of mass of $\mathrm{H}_{2}\left(\mathrm{D}_{2}\right)$ and glyoxal. The function $\varphi(r)$ is the solution of the Hamiltonian describing the vibration of the uncomplexed glyoxal molecule, while $\theta(R)$ is the eigenfunction describing the van der Waals motion when the glyoxal molecule is "frozen" at its equilibrium geometry $r_{e}$. In the distorted wave treatment of vibrational predissociation, the individual rates are given by ${ }^{1}$

$$
\Gamma_{v \rightarrow v^{\prime}}=\pi\left|<\theta^{\text {(bound) }} \varphi_{v}\right|\left(V(R, r)-V\left(R, r_{e}\right)\right) \mid \theta^{(\text {diss })} \varphi_{v^{\prime}}>1^{2}
$$

where $V(R, r)$ is the van der Waals intermolecular potential. In Eq. (2), $v$ denotes the initial vibrational state of the glyoxal molecule in the bound state of the complex, while $v^{\prime}$ is the final vibrational state of the glyoxal molecule after dissociation. The branching ratio between two different dissociation pathways $v^{\prime}$ and $v^{\prime \prime}$ obtained from the same initial state $v$ will then be given by

$$
R\left(v^{\prime} / v^{\prime \prime}\right)=\frac{\Gamma_{v \rightarrow v^{\prime}}}{\Gamma_{v \rightarrow v^{\prime \prime}}}
$$

If the van der Waals interaction potential $V(R, r)$ is described in terms of a sum of atom-atom interactions, and if the potential at the equilibrium geometry of the glyoxal $V\left(R, r_{e}\right)$ is fitted by a Morse function

$$
V\left(R, r_{e}\right)=D\left\{\exp \left[-2 \alpha\left(R-R_{o}\right)\right]-2 \exp \left[-\alpha\left(R-R_{o}\right)\right]\right\},
$$

it has been shown ${ }^{1}$, that the coupling term $\left[V(R, r)-V\left(R, r_{e}\right)\right]$ in Eq. (2) can be given approximately by $A \exp (-2 \alpha R)$, the proportionality constant A depending on the complex geometry and on the decomposition of the normal coordinates on the cartesian displacements. If we formed the ratio $y=R_{H_{2}} / R_{D_{2}}$, this constant cancels and $y$ is function only of the potential parameters $\alpha, D$ and of the relative kinetic energy of the fragments as well 


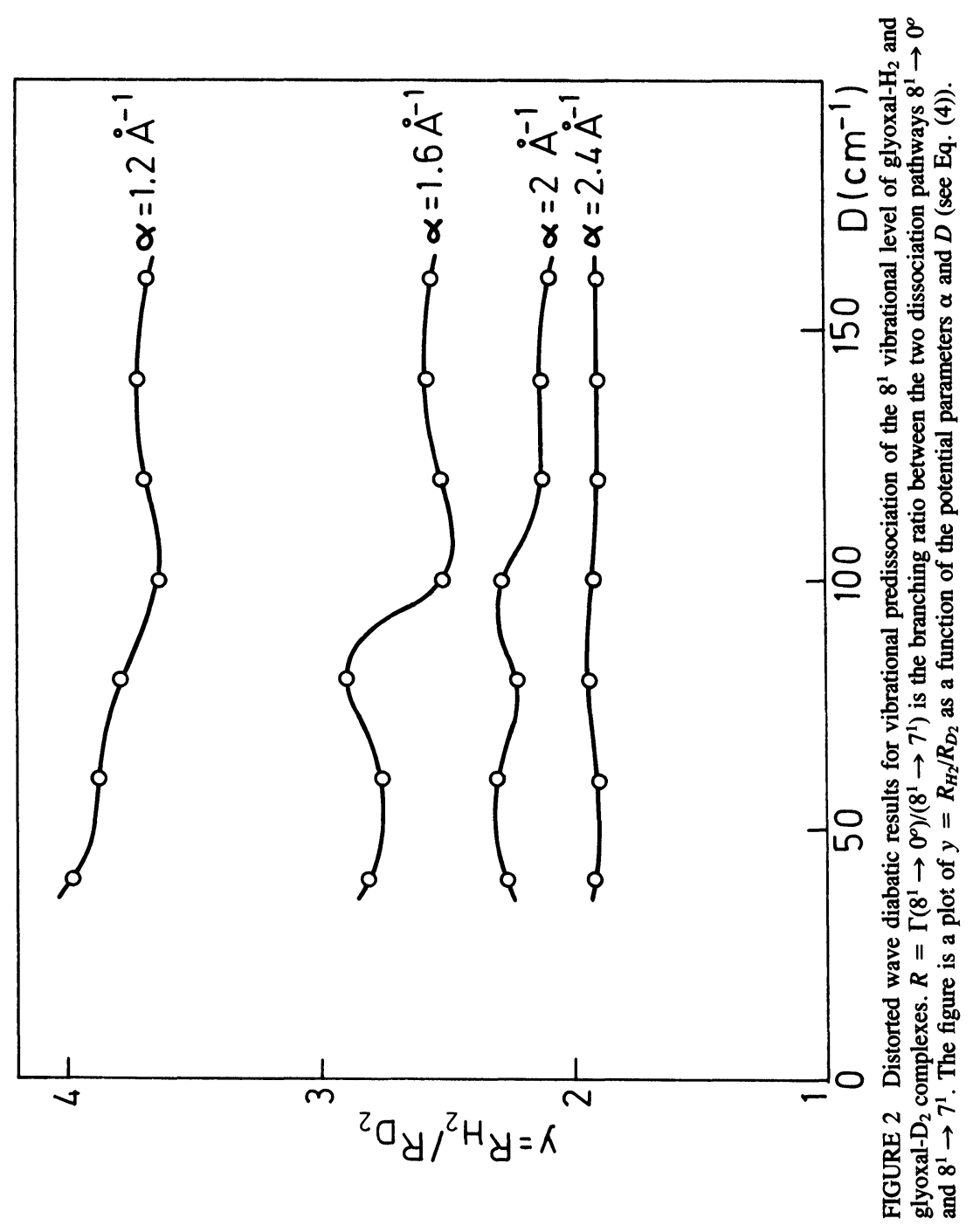


as of the reduced mass for the relative motion of $\mathrm{H}_{2}\left(\mathrm{D}_{2}\right)$ with respect to the glyoxal molecule.

We have performed calculations using the analytical results developed for Morse functions ${ }^{1}$. The parameters and $D$ were estimated in the range $1 \AA^{-1}<\alpha<3 \AA^{-1}$ and $50 \mathrm{~cm}^{-1}<D<150 \mathrm{~cm}^{-1}$. The results for the vibrational predissociation of the $8^{1}$ state of glyoxal giving $7^{1}$ and $\mathrm{O}^{\circ}$, are displayed on Figure 2.

It can be seen that $y$ is always greater than 1 and varies from 2 to 4 for the range of parameters chosen. These results agree with the experimental finding that $y>2.5$.

The reason why $y$ is always greater than 1 in our calculations is the following: the rates are proportional to the overlap between an initial bound wavefunction and a continuum dissociative function. The former has a width proportional to $\alpha^{-1}$ while the latter oscillates with a wavelength $\lambda$ $=(2 \mu \varepsilon / \hbar)$ where $\varepsilon$ is the relative kinetic energy. The rates will then be a strong increasing function of the product $\lambda \alpha$. From our analytical results, it is possible to show $^{1}$ that $\Gamma \propto \exp (-\pi / \lambda \alpha)$. Now $\lambda$ increases when $\mathrm{H}_{2}$ is substituted by $D_{2}$ because the reduced mass increases, but it is also larger for the $8^{1} \rightarrow \mathrm{O}^{\circ}$ than for the $8^{1} \rightarrow 7^{1}$ dissociation process. As a result, the branching ratio between the final states $\mathrm{O}^{\circ}$ and $7^{1}$ is greater for $\mathrm{H}_{2}$ than for $\mathrm{D}_{2}$ in agreement with the experimental results. Similar isotope effects should be present for other initial states in glyoxal as well as in other molecular systems such as $\mathrm{I}_{2}-\mathrm{H}_{2}\left(\mathrm{D}_{2}\right)^{8}$.

\section{Acknowledgment}

We would like to acknowledge Dr D. A. Ramsay for suggesting this study.

\section{References}

1. J. A. Beswick and J. Jortner in Photoselective Chemistry, Adv. Chem. Phys. 47, 363 (1981).

2. D. H. Levy in Photoselective Chemistry, Adv. Chem Phys. 47, 323 (1981).

3. N. Halberstadt and B. Soep, Chem. Phys. Lett. 87, 109 (1982).

4. N. Halberstadt, Thesis, Université Paris-Sud, 1982.

5. J. A. Beswick, N. Halberstadt and B. Soep, to be published.

6. C. Jouvet and B. Soep, J. Chem. Phys., 75, 1661 (1981).

7. N. Halberstadt and J. A. Beswick, Faraday Disc. $\mathrm{n}^{\circ} 73$ (1982), in press.

8. J. E. Kenny, T. D. Russell and D. H. Levy, J. Chem. Phys. 73, 3607 (1980). 